\title{
CAN VERY LARGE-SCALE STRUCTURES EXIST IN THE UNIVERSE?
}

\author{
J. M. Martǐn-Mirones and L. J. Goicoechea \\ Departamento de Fïsica Moderna \\ Universidad de Cantabria \\ Avda. de Los Castros s/n \\ 39005 Santander, Cantabria Spain
}

\section{INTRODUCTION}

The controversy about the position of the matter accumulation that causes our peculiar motion with respect to the cosmic background radiation (CBR) has very recently acquired great interest. So, the very local attractor suggested by Lynden-Bell et al. (1988) and Dressler (1988) begins to be questioned. The histogram done by Melnick and Moles (1987) shows that the dominant structure may be at $\sim 140 h^{-1} \mathrm{Mpc}$. Similar results have been obtained by Scaramella et al. (1989). On the other hand, the analysis of the very local candidate in the Xray band shows that this does not have rich clusters of galaxies (Jahoda and Mushotzky 1989). Moreover, Lahav et al. (1989) have studied a sample of 53 clusters emitting X-rays $(E \approx 2-20$ $\mathrm{keV}, L_{X} \approx 5 \times 10^{42}-7 \times 10^{44} h^{-2} \mathrm{erg} \mathrm{s}^{-1}$ ); they have observed a great concentration of clusters in the direction of the attractor suggested by Lynden-Bell et al., but at a distance of $\sim 100$ $h^{-1}-150 h^{-1} \mathrm{Mpc}$. The detections of nonlocal structures (further than $300 h^{-1} \mathrm{Mpc}$ ) are very few. However, the study done by Shaver (1987) using catalogs of quasars showed that a superstructure with a radius of $-200 h^{-1} \mathrm{Mpc}$ and with a density in excess of $\delta \approx 1$ may exist at a distance of $\sim 800 \mathrm{~h}^{-1} \mathrm{Mpc}$.

It is important to analyze the observational effects of these very large-scale $\left(\geq 100 h^{-1}\right.$ Mpc) structures (which challenge the modern theories about the origin and the characteristics of the galaxy accumulations). Unfortunately, the effect on the magnitude/redshift relation is not observable due to the great errors made when the absolute magnitudes are determined (Goicoechea and Martin-Mirones 1989a). The effects on the CBR (microwaves and X-rays) are more interesting. Here we show the main results that we have obtained.

\section{RESULTS AND DISCUSSION}

If we assume that the superstructures (scale $\gtrsim 10^{2} h^{-1} \mathrm{Mpc}$ ) exist since the epoch of the decoupling of the matter and the radiation and that they are spatially uncorrelated (Poissonian distribution), we can use the observational limit $(\Delta T / T)_{10^{\circ}}$ (where $T$ is the temperature of the microwave background radiation and $(\Delta T / T)_{10^{\circ}}$ is its anisotropy on an angular scale of $\sim 10^{\circ}$ ) to obtain the probability that the dipole anisotropy of $T$ is due to a certain structure (Goicoechea and Martin-Mirones 1989a). So, the probability that our attractor is the accumulation suggested by Melnick and Moles (1987) is $\sim 2.7 \%$, whereas the probability that such an attractor is the accumulation suggested by Shaver (1987) is $\sim 2 \%$. In this calculation, we do not consider three effects that may change the results moderately or strongly: the width of the antennae, the possible existence of great voids, and the possible correlations among the 
superstructures.

We have also studied the direct effect of the superstructures on the X-ray background (intensity excess in the direction of the structure due to the accumulation of sources in the region where the structure is placed) by using the observational information about the relative difference between the mean intensity coming from the hemisphere determined by the attractor and the mean intensity coming from the opposite hemisphere; the value of this anisotropy has been obtained by means of the data from the Ariel $V$ satellite. We consider attractors with different geometrical forms (spheres, disks, and filaments) that are immersed in different populations of X-ray (2-20 keV) emitters. The luminosity function of the real population of emitters is not known. However, the AGNs are the main type of known sources that contribute to the X-ray background (XRB). So, recent studies show that the population of AGNs with low intrinsic luminosity $\left(L_{X} \leq 10^{43} \mathrm{erg} \mathrm{s}^{-1}\right.$ ) does not evolve with the redshift (Danese et al. 1986). If we consider that these objects, having no cosmological evolution, are a good tracer of the matter distribution, the existence of an attractor at $\sim 150 h^{-1} \mathrm{Mpc}$ in a flat universe is only compatible with a contribution to the XRB $f x<20 \%$ (spherical attractor) or $a<12 \%$ (filament with moderate eccentricity). If the attractor is flattened (disk), the contribution of the AGNs with low luminosity to the XRB can reach a value of $\sim 30 \%$. For a nonlocal inhomogeneity (like that suggested by Shaver (1987)), the maximum possible contribution is $\sim 40 \%$. Therefore, the hypothesis that the AGNs with low luminosity generate most of the XRB is incompatible with the existence of a very large-scale attractor (Goicoechea and Martin-Mirones 1989b).

Finally, we must notice that the existence of the very large-scale attractor suggested by Melnick and Moles (1987), Lahav et al. (1989), and Scaramella et al. (1989) implies the following (by using physically reasonable models and observations on the anisotropy of the CBR):

1. There exist large empty regions $\left(\delta=-1\right.$ on scales $\left.\gtrsim 10^{2} h^{-1} \mathrm{Mpc}\right)$ in the universe.

2. The contribution of the low luminosity AGNs to the XRB is $f x<12-30 \%$.

3. The relative brightness excess of the XRB on scales of a few degrees in the direction of the center of the attractor (due to the accumulation of AGNs) is less than $2 \%$ (we assume that the bright AGNs cannot generate more than $15 \%$ of the XRB according to the estimations by Kembhavi and Fabian (1982) and Barcons and Fabian (1988)).

\section{REFERENCES}

Barcons, X. and Fabian, A. C. 1988, MNRAS, 230, 189.

Danese, L. et al. 1986, Astr. Ap., 161, 1.

Dressler, A. 1988, Ap. J., 329, 519.

Goicoechea, L. J. and Martin-Mirones, J. M. 1989a, Astr. Ap., in press.

Goicoechea, L. J. and Martin-Mirones, J. M. 1989b, preprint.

Jahoda, K. and Mushotzky, R. F. 1989, Ap. J., in press.

Kembhavi, A. K. and Fabian, A. C. 1982, MNRAS, 198, 921.

Lahav, O. et al. 1989, MNRAS, in press.

Lynden-Bell, D. et al. 1988, Ap. J., 326, 19.

Melnick, J. and Moles, M. 1987, Rev. Mexicana Astr. Astrof., 14, 72.

Scaramella, R. et al. 1989, Nature, 338, 562.

Shaver, P. 1987, Nature, 326, 773. 